\title{
Viscoelastic Mapping of the Arterial Ovine System using a Kelvin Model
}

\author{
D. Valdez-Jasso ${ }^{1}$, M.A. Haider ${ }^{1}$, H.T. Banks ${ }^{1}$, D. Bia ${ }^{2}$, Y. Zocalo ${ }^{2}$, \\ R. Armentano ${ }^{2}$, and M.S. Olufsen ${ }^{1}$ (msolufse@ncsu.edu)
}

\author{
1) Department of Mathematics and \\ Center for Research in Scientific Computation \\ North Carolina State University \\ Campus Box 8205 \\ Raleigh, NC 27695. \\ 2) Department of Physiology \\ School of Medicine \\ Universidad de la Republica \\ General Flores 2125, PC: 11800 \\ Montevideo, Uruguay.
}

March 19th, 2007

\begin{abstract}
The mechanics of the arterial wall is complex, due to its material structure and load conditions, which influence the hemodynamic properties as well as the growth and remodeling process of the cardiovascular system. Arterial remodeling can be found both locally and globally. Local remodeling is typically a result of disease, while global remodeling can be found even for healthy arteries. In this study we have analyzed how elastic and viscoelastic properties differ across 7 locations along the large ovine arteries in 11 sheep. We combined the Kelvin model with experimental measurements of vessel diameter and pressure obtained in-vitro at conditions mimicking the in-vivo dynamics. Elastic and viscoelastic wallproperties were assessed by analyzing values of four model parameters across the 7 locations. To do so we solved an inverse problem, resulting in computed estimates for each of the four parameter values that minimize the residual between the data and the model. We used sensitivity analysis to compute standard errors, and confidence intervals for all model parameters. Results showed that while elastic properties including Young's modulus and the vessel wall thickness varied across locations (smaller arteries were stiffer than larger arteries) viscoelastic relaxation parameters did not differ significantly across locations. We also showed that for all locations, the inclusion of viscoelastic behavior, e.g., using the Kelvin model, is important to capture pressure-area dynamics.
\end{abstract}

\section{INTRODUCTION}

The mechanics of the arterial wall is complex, due to its material structure and load conditions, which influence the hemodynamic properties as well as the growth and remodeling process of the cardiovascular system [1]. Remodeling can be found both locally and globally. Local remodeling is often the result of disease, while global remodeling can be found even within healthy subjects. In particular, it is well known that the composition of collagen, smooth muscle, and elastin vary along the vessels. Large arteries are more distensible, thus they are better suited to dampen some of the pulsatile response, while smaller 
arteries are stiffer, but contain more collagen and smooth muscle fibers allowing them to constrict or dilate to regulate blood flow [2]. One study [3], which examined wall properties in situ showed that peripheral vessels (in particular femoral and carotid arteries) were stiffer than the abdominal aorta. This was demonstrated experimentally, e.g., [3], by comparing simultaneous measurements of blood pressure to the relative change in the vessel diameter. They determined the vessel distensibility by calculating the slope of the diameter/pressure curve. While this apparent quantity gives some measure of compliance, it does not provide any detailed information about Young's modulus or viscoelastic properties of the vessel wall, which we will consider by direct analysis of the experimental data using a viscoelastic mathematical model.

Another important observation is that the mechanical properties of arteries are further modified by aging and/or disease (e.g., hypertension or atherosclerosis). In particular responses to hypertension have been studied extensively [2], [4], [5], [6], [7], [8], [9]). These changes in wall properties occur both locally and globally. Typical local changes can be traced in areas prone to atherosclerosis, whereas global changes can be observed in patients suffering from hypertension or diabetes.

The arterial wall is complex, it contains several distinct layers, and each of these layers (the intima, the media, and the adventitia) play a role in determining the overall mechanics of the wall. Detailed studies of the microscopic mechanics of each of these layers have been described in work by Humphrey [10] and Holzapfel [11], [12]. These studies showed that the intima, the innermost layer, is a single endothelial cell layer, and ensures that blood does not adhere to the wall. This layer is responsible for some of the local biophysical control mechanisms, but it does not contribute significantly to the passive arterial wall mechanics. Instead, the two outer layers, the media and adventitia, play the most important role in determining mechanical properties of the vessels under physiological flow conditions. The basic components of these layers are smooth muscle cells, elastin, collagen, proteoglycans, nerves, and fibroblasts. Together, these components make the vessels display both elastic and viscoelastic mechanical behavior [2], [13], which will be studied in this work.

Improved knowledge of the mechanical properties of the vessel walls can also be used in the design of better fluid dynamics models developed to predict flow and pressure in the systemic arteries. Typically these models need some description of the dynamics of the arterial wall. Thus, if a more advanced viscoelastic wall mechanics model can be developed, it may be of interest to researchers developing fluid dynamic models, particularly if the wall mechanics model can be easily integrated into the fluid solver. At present, most fluid mechanics models (e.g., [14], [15], [16], [17], [18], [19], [20], [21], [22]), assume either that arteries are rigid or exhibit a purely elastic response. While more recent studies [23], [24], [25], [26], have accounted for viscoelastic properties, they have not incorporated variations in wall properties at different locations in the network. Consequently, a mathematical viscoelastic model considering the regional differences among system arteries remains to be validated.

The goal of this study is to explore how a simple viscoelastic model such as the Kelvin model can be used to predict simultaneous measurements of cross-sectional area and arterial blood pressure from 7 different locations in sheep arteries. In particular, we have studied how the elastic and viscoelastic properties are modified across vessels proximal and distal to the heart. To study this, we have developed a mathematical model that relates blood pressure and cross-sectional area, and validated this model against data obtained from eleven Merino sheep. Using measured pressure data as an input, we used nonlinear optimization to compute viscoelastic model parameters that minimized the difference between computed and measured values of the cross-sectional area. With this optimization we obtained a high coherence between our model and the data and, we demonstrated that the viscoelastic model captures essential features of the data significantly better than a traditional elastic wall model. This study also showed that the elastic moduli change along the vessels, and in particular, we showed that peripheral vessels are more rigid than proximal vessels, while the viscoelastic relaxation times cannot be distinguished across the seven sites. 


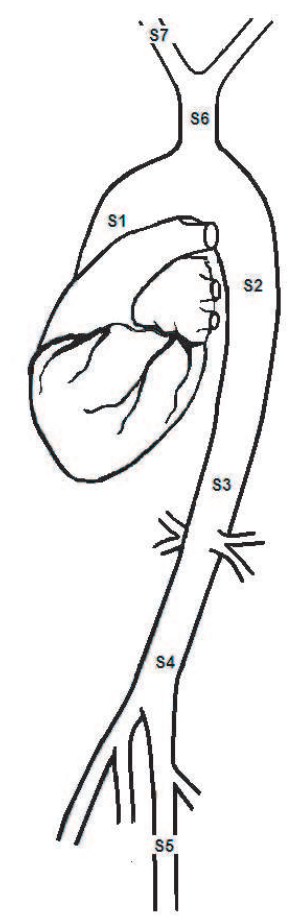

Fig. 1. Scheme of the studied arterial segments. S1: ascending aorta; S2, S3 and S4: proximal, medial, and distal descending aorta; S5: femoral artery; S6: brachiocephalic trunk; S7: carotid artery.

\section{Methods}

\section{A. Experimental methods}

1) Surgical preparation and segment's acquisition: Eleven healthy male Merino sheep, weighing 25 to $35 \mathrm{~kg}$, were included in this study. All protocols were approved by the Research and Development Council of the Universidad de la Republica, and were conducted in accordance with the Guide for the Care and use of Laboratory Animals [27].

All animals were vaccinated and treated for skin and intestinal parasites. During 30 days before surgery, they were appropriately fed, and assessed for optimal clinical status. General anesthesia was induced with intravenous administration of pentobarbital $(35 \mathrm{mg} / \mathrm{kg})$. The respiration was maintained with a positive respirator (Dragger SIMV Polyred 201, Madrid, Spain). Respiratory rate, tidal volume, and the inspired oxygen fraction were adjusted to maintain arterial $\mathrm{pCO}_{2}$ at $35-45[\mathrm{mmHg}], \mathrm{pH}$ at 7.35-7.4, and $\mathrm{pO}_{2}$ above 80 [mmHg].

Eleven arteries, from different regions were selected in order to evaluate their biomechanical properties: carotid, brachiocephalic trunk, ascending aorta, proximal, medial and distal descending aorta, and femoral artery, see Fig. 1. Arteries were exposed and dissected, and a $6[\mathrm{~cm}]$ length segment of each artery was in situ measured and marked with two suture references in the adventitia. In order to measure the external diameter, a pair of ultrasonic crystals $(5[\mathrm{MHz}], 2[\mathrm{~mm}]$ diameter) was sutured to the adventitia (on opposite sites). The transit time of the ultrasonic signal $(1580[\mathrm{~m} / \mathrm{s}])$ between the crystals was converted into distance (arterial external diameter) by means of a sonomicrometer $(1000 \mathrm{~Hz}$ frequency response, Triton Technology Inc. San Diego, CA, USA). According to the specifications of the Sonomicrometer Operator Manual (Triton Technologies), the diameter signal was calibrated in [mm] using the sonomicrometer $1[\mathrm{~mm}]$ step calibration facility. Optimal positioning of the dimensional gauges was assessed by an oscilloscope (model 465B, Tektronix, Richardson, Texas, USA), according to the specifications of the sonomicrometer Operator Manual (Triton Technologies, San Diego, CA, USA). 
After that, the animals were sacrificed with an intravenous overdose of pentobarbital followed by potassium chloride. The correct position of the ultrasonic crystals was confirmed at necropsy. All procedures were performed like in previous work [2], [28], [29], [30], [31], [32], [33], [34], [35].

2) In vitro studies: The segments were non-traumatically mounted (at in vivo length) in the organ chamber of the circulation mock; immersed and perfused with a thermally regulated $\left(37^{\circ} \mathrm{C}\right)$ and oxygenated Tyrode's solution, with $\mathrm{pH}$ equal 7.4, see Fig. 2. The circulation mock, consisted in polyethylene tubing powered by a pneumatic pump (Jarvik Model 5, Kolff Medical Inc., Salt Lake City, Utah, USA). The pneumatic device was regulated by an air supply machine that allowed adjustments of hemodynamic parameters values and waveforms. More details related to the circulation mock are available in previous articles [28], [29], [33], [34], [35], [36]. Pressure was measured with a solid-state micro transducer (1200

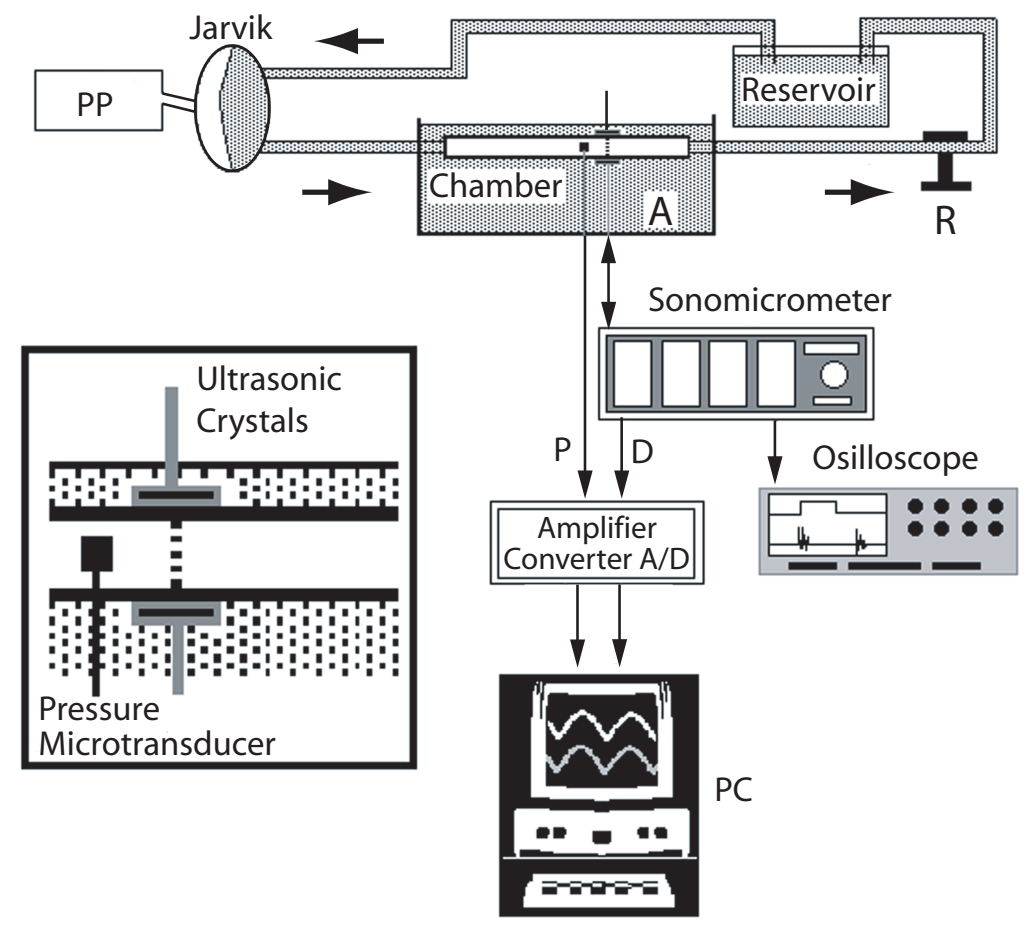

Fig. 2. Circulating mock showing a pneumatic pump (PP) and a perfusion line with a chamber, a resistance modulator (R) and a reservoir. Tyrode's solution is thermally controlled (A). The pressure signal (P) is obtained using a solid transducer, and the diameter (D) signals are obtained using a pair of ultrasonic crystals and a sonomicrometer. All signals are monitored on an oscilloscope and stored in a personal computer (PC).

$[\mathrm{Hz}]$ frequency response, Konigsberg Instruments, Inc., Pasadena, CA, USA), inserted in each artery through a little incision. Pressure sensors were previously calibrated using a mercury manometer. To avoid signals interference, the pressure sensor was inserted 2 [mm] proximal to the diameter crystals [31], a distance carefully measured, taking into account its importance for an adequate signal analysis [31], [37]. Finally, a non-constricting ultrasonic perivascular flow probe (Model T206, Transonic Systems Inc., Ithaca, NY, USA) was positioned around each artery. The technique described has been used by members of our group both in in vivo [2], [30], [31], [32] and in vitro studies [28], [29], [33], [34], [35].

Once placed in the organ chamber, the segments were allowed to equilibrate for a period of 10 minutes under a steady state of flow and pressure at a stretching rate of 1.8 [Hz] $(108$ c.p.m). Then, the segments were submitted to physiological hemodynamic conditions [31], [32], [36-38]. The segments' pressure and diameter signals were displayed in real time, digitized every 5 [ms] and stored for later analysis. Approximately 10 consecutive cardiac cycles were sampled and analyzed. 


\section{B. Mathematical Modeling}

Two continuum models were used to study the physical behavior of the arterial wall: an elastic model and a viscoelastic model.

Starting with the elastic model, we formulated a stress-strain relation using Hooke's law based on work by Fung [38], [39]. To determine stress, the artery was idealized as a thin walled tube, with circumferential stress defined as $\sigma_{\theta \theta}=p(t) r(t) / h$. The $\sigma_{\theta \theta}$ stress term represents the average stress along a cross-section of the arterial wall. In this equation, $h[\mathrm{~cm}]$ is the thickness of the wall, $p(t)[\mathrm{mmHg}]$ is the pressure and $r(t)[\mathrm{cm}]$ is the radius of the vessel.

Assuming that the axial length and tension on a cross-section are constant and obey Hooke's Law, the circumferential strain is given by $e_{\theta \theta}=\left(\sigma_{\theta \theta}-v \sigma_{r r}\right) / E$, where $E[\mathrm{mmHg}]$ is Young's modulus and $v$ is Poisson's ratio. Because of the thin wall approximation, $\sigma_{r r} \ll \sigma_{\theta \theta}$; hence $\sigma_{r r}$ can be neglected. Consequently, the strain $e_{\theta \theta}$ is then equal to the change of radius divided by the radius of the unstretched vessel $r_{0}$

$$
e_{\theta \theta}=\frac{r-r_{0}}{r_{0}}
$$

which gives

$$
r(t)=\frac{e_{\theta \theta} E h}{p(t)}=\frac{E h\left(r(t)-r_{0}\right) / r_{0}}{p(t)} \Leftrightarrow p(t)=\frac{E h}{r_{0}}\left(1-\sqrt{\frac{A_{0}}{A}}\right),
$$

where the cross-sectional area of the artery $A(t)=\pi r(t)^{2}\left[\mathrm{~cm}^{2}\right]$.

The elastic model in Equation (1) can be written in a quasi-static stress-strain form

$$
p(t)=\frac{E h}{r_{0}} s(t), \quad s(t)=\left(1-\sqrt{\frac{A_{0}}{A(t)}}\right) \quad \Leftrightarrow \quad A(t)=\frac{A_{0}}{(s(t)-1)^{2}},
$$

where the stress is the pressure $p(t)$, and the strain $s(t)$ is a measure of relative change in the crosssectional area, $A(t)$. However, it is well known that the arterial wall possesses a viscoelastic behavior [40]; thus, we extend the stress-strain relation in Equation (2) to a viscoelastic model of flow-induced arterial wall deformation. The Kelvin model idealizes the behavior of a viscoelastic material using a linear

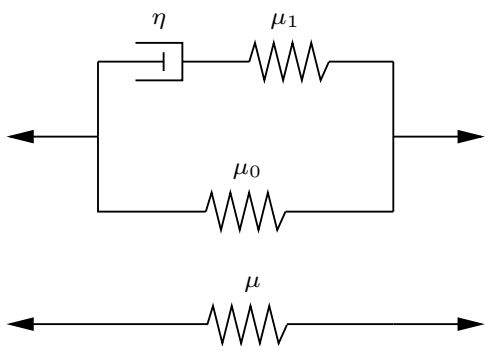

Fig. 3. The Kelvin model is often displayed using an electrical circuit analog with a dashpot acting on a spring (top) incorporates the time dependent viscosity property, in parallel with a spring (bottom), representing the elastic component of the material.

combination of springs and a dashpot in a mechanical circuit to represent elastic and viscous components respectively as shown in Fig. 3. Mathematically, the stress-strain law for this viscoelastic model can be written as

$$
\tau_{\epsilon} \frac{d s}{d t}+s=\frac{r_{0}}{E h}\left(p+\tau_{\sigma} \frac{d p}{d t}\right)
$$

where $r_{0}$ is the radius of the unstretched vessel, and $s(t)$ and $p(t)$ are the strain and stress (represented by pressure). In Equation (3), $\tau_{\epsilon}$ is a relaxation time for the strain and $\tau_{\sigma}$ is a relaxation time for the stress. The two relaxation constants are related to the dashpot and resistors as follows

$$
\tau_{\sigma}=\frac{\eta}{\mu_{1}}, \quad \tau_{\epsilon}=\frac{\mu_{1}}{\mu_{0}}\left(1+\frac{\mu_{0}}{\mu_{1}}\right), \quad \mu_{0}=\frac{E h}{r_{0}} .
$$


When solving the viscoelastic model, which has the implicit form

$$
F(s(t), \dot{s}(t), p(t), \dot{p}(t), \theta)=0,
$$

we chose to solve Equation (3) in terms of the strain $s(t)$ as a function of time, pressure history and the parameters in study, i.e.,

$$
s(t)=s\left(t, p_{t}(\cdot), \theta\right), \text { where } \theta=\left(E h, r_{0}, \tau_{\sigma}, \tau_{\epsilon}\right) .
$$

The notation $p_{t}(\cdot)$ is defined by

$$
p_{t}(\cdot)=\{p(\xi) \mid 0 \leq \xi \leq t\} .
$$

Note that this representation includes the hysteresis effects known to be present in viscoelastic materials.

To solve the viscoelastic model in Equation (3) numerically, it is advantageous to write the model as an integral of the form is given by

$$
s(t)=s(0) e^{-t / \tau_{\epsilon}}+\frac{r_{0}}{E h \tau_{\epsilon}}\left(\tau_{\sigma} p(t)-\tau_{\sigma} p(0) e^{-t / \tau_{\epsilon}}+\frac{\tau_{\epsilon}-\tau_{\sigma}}{\tau_{\epsilon}} \int_{0}^{t} e^{-(t-\gamma) / \tau_{\epsilon}} p(\gamma) d \gamma\right) .
$$

From this equation, the area $A$ at time $t$ can then be expressed as a function of the pressure history using the definition for strain $s(t)$ as a function of $A(t)$ introduced in the center of Equation (2).

The Kelvin viscoelastic model is preferred over the simpler Maxwell and Voigt models since it is the simplest model that exhibits both creep and stress relaxation. While more advanced models can be considered via a superposition Kelvin units, the approach in this study is to apply the simplest possible model that captures fundamental viscoelastic properties of arterial wall in the analysis of a comprehensive experimental data set for flow-induced viscoelastic arterial wall deformation.

1) Model validation: The model was validated using experimental data from eleven sheep as described previously. The data include in-vitro pressure and vessel diameter from seven locations along the large arteries. In the following we denote pressure by $\left\{p_{j}\right\}$ and arterial diameters by $\left\{d_{j}\right\}$, both of these quantities are sampled at times $t_{j}$ at a frequency of $20 \mathrm{~Hz}$. Approximately 10 consecutive cardiac cycles were sampled and analyzed for each sheep. It is important to note that the measured values $p_{j}$ and $d_{j}$ provide information about vessel pressure and diameter, respectively, and the measured cross-sectional area values were obtained by $a_{j}=\pi\left(d_{j} / 2\right)^{2}$.

For our modeling study we have chosen to interpolate the measured pressure $\left\{p_{j}\right\}$ and use this interpolation $\{\tilde{p}(t)\}$ in (7) as a model input to predict cross-sectional area $\left\{A\left(t_{j}\right)\right\}$ as a function of the parameters $\theta$ via Equations (2) and (7). The Kelvin viscoelastic model in Equation (3) relates vessel strain to vessel stress (pressure), and involves the cross-sectional area via the definition of strain in Equation (2). This particular definition of strain was obtained such that the Kelvin model reduced to the simpler elastic model when the relaxation times are set to zero. We remark that we could have alternatively expressed the pressure $p(t)$ as a function of the history $s_{t}(\cdot)$ of the strain, leading through Equation (2) to the pressure $p(t)$ at time $t$ as a function of the history $A_{t}(\cdot)$ of the area.

The initial parameter values for $E h$ in Table I were obtained from Fung's estimates of wall thickness $h$ and Young's modulus $E$ [38], [39]. It should be noted that in principle $E$ and $h$ are two independent parameters, but in this study we have no data to estimate these separately, thus they have been combined into one parameter. We do not have significant knowledge about the relaxation factors $\tau_{\sigma}$ and $\tau_{\epsilon}$. We assumed that they may be an order apart, thus we estimated the same initial values for all locations in the system. Initial values for the final parameter $r_{0}$ was estimated for each sheep using an average diastolic diameter from the area measurements.

However, it is known that the morphometry and tissue composition varies significantly among subjects and among the locations in the arterial system. Hence, to obtain "subject" specific parameters we used the nonlinear Nelder-Mead optimization method (a simplex method) to compute parameters that minimize 
TABLE I

INITIAL PARAMETER VALUES FOR $E h, \tau_{\sigma}$, AND $\tau_{\epsilon}$ IN HUMANS.

\begin{tabular}{l|rrr} 
& $E h[\mathrm{mmHg} \mathrm{cm}]$ & $\tau_{\sigma}[\mathrm{sec}]$ & $\tau_{\epsilon}[\mathrm{sec}]$ \\
\hline Ascending aorta (S1) & 234.200 & 0.025 & 0.05 \\
Proximal descending aorta (S2) & 180.153 & 0.025 & 0.05 \\
Medial descending aorta (S3) & 337.787 & 0.025 & 0.05 \\
Distal descending aorta (S4) & 375.319 & 0.025 & 0.05 \\
Femoral artery (S5) & 300.255 & 0.025 & 0.05 \\
Brachiocephalic trunk (S6) & 234.199 & 0.025 & 0.05 \\
Carotid artery (S7) & 202.672 & 0.025 & 0.05
\end{tabular}

the least squares error between the computed and measured values of cross-sectional area. The least squares error is given by

$$
J=\frac{1}{n} \sum_{i}^{n}\left|a_{j}-A\left(t_{j}\right)\right|^{2} .
$$

It is important to note that even though this method is used to solve modeling problems, it is not a proper least squares problem. Our prediction of the cross-sectional area $A\left(t_{j}\right)$ based on the Kelvin viscoelastic model used measured pressure values $\left\{p_{j}\right\}$ as an input. These values $\left\{p_{j}\right\}$ were interpolated and then integrated to obtain values for the first strain, and subsequently provided computed values for crosssectional area $A\left(t_{j}\right)$. In other words, we are integrating nonexact (noisy) pressure values obtained from $\left\{p_{j}\right\}$, which gives rise to noisy predictions of cross-sectional area $\left\{A\left(t_{j}\right)\right\}$. Typical results of fitting the viscoelastic model to the data using Equation (8) are shown in Fig. 4 and summarized in Table II. From Fig. 4, it is clear that the viscoelastic model captures the dynamics significantly better than the elastic model. In particular, the difference between the models is emphasized in the graph where area is plotted against pressure, where the data shows a hysteresis loop, a characteristic of a viscoelastic material. Given that the elastic model describes a proportional relationship with no relaxation factors, it yields a straight line response that is unable to exhibit hysteresis. For a numerical representation, refer to Table II that lists the optimized values found for the parameter in study, and Table III for the mean values and standard deviations across the complete dataset. Without further analyzing the model, it is not clear whether it is possible to uniquely identify all the parameters of the model given that pressure is the input for prediction of cross-sectional area. One way to investigate if all four model parameters can be identified is by a sensitivity analysis, which determines if the model parameters are sensitive to changes in cross-sectional area.

Sensitivity equations can be obtained using the basic differential equation analysis approach as described by Eslami and Frank [41], [42]. For the Kelvin model there is only one state $A(t, \theta)$ and four parameters denoted by $\theta=\left(\theta_{1}, \ldots, \theta_{4}\right)=\left(r_{0}, E h, \tau_{\epsilon}, \tau_{\sigma}\right)$. In other words, sensitivities (absolute) provide information about the change in the state variable $A(t)$ with respect to each of the four parameters. Mathematically, these absolute sensitivities $S_{j}$ can be defined by

$$
S_{j}(t, \theta)=\frac{\partial A(t, \theta)}{\partial \theta_{j}}, \quad j=r_{0}, E h, \tau_{\epsilon}, \tau_{\sigma} .
$$

The absolute sensitivities defined above may lead to wrong conclusions regarding the degree of dependence on various parameters. In particular, if the parameters have different orders of magnitude as is the case for Kelvin's viscoelastic model. Therefore, it is important to compute sensitivities that are normalized by the nominal outputs and the parameter values as follows

$$
S_{j}(t, \theta)=\frac{\theta_{j}}{A(t, \theta)} \frac{\partial A(t, \theta)}{\partial \theta_{j}} .
$$

It is easily seen that the relative sensitivities are invariant of changes in units of $A(t)$ or $\theta$. This allows the comparison of sensitivities across different parameter values. 
TABLE II

OPTIMIZED PARAMETER VALUES FOR ALL SHEEP. FIRST COLUMN IS THE SHEEP \#, SECOND COLUMN $r_{0}$ [CM], THIRD COLUMN Eh [MMHG CM], FOURTH COLUMN $\tau_{\epsilon}$ [SEC] AND THE LAST COLUMN $\tau_{\sigma}$ [SEC].

\begin{tabular}{|c|c|c|c|c|c|c|c|c|c|c|c|c|c|c|}
\hline$\#$ & $r_{0}$ & $E h$ & $\tau_{\epsilon}$ & $\tau_{\sigma}$ & $\#$ & $r_{0}$ & $E h$ & $\tau_{\epsilon}$ & $\tau_{\sigma}$ & $\#$ & $r_{0}$ & $E h$ & $\tau_{\epsilon}$ & $\tau_{\sigma}$ \\
\hline \multicolumn{5}{|c|}{ Ascending aorta } & \multicolumn{5}{|c|}{ Brachiocephalic trunk aorta } & \multicolumn{5}{|c|}{ Carotid artery } \\
\hline 1 & 0.91 & 468.771 & 0.05 & 0.02 & 1 & 0.803 & 508.038 & 0.067 & 0.027 & 1 & 0.450 & 2939.569 & 0.105 & 0.044 \\
\hline 2 & 1.02 & 646.974 & 0.06 & 0.02 & 2 & 0.825 & 487.788 & 0.153 & 0.061 & 2 & 0.404 & 3267.155 & 0.076 & 0.037 \\
\hline 3 & 1.01 & 545.324 & 0.09 & 0.04 & 3 & 0.816 & 527.147 & 0.066 & 0.021 & 3 & 0.395 & 3026.771 & 0.033 & 0.007 \\
\hline 4 & 0.97 & 540.081 & 0.07 & 0.02 & 4 & 0.876 & 638.347 & 0.063 & 0.016 & 4 & 0.436 & 3160.495 & 0.108 & 0.063 \\
\hline 5 & 0.97 & 596.347 & 0.07 & 0.03 & 5 & 0.855 & 670.538 & 0.058 & 0.016 & 5 & 0.410 & 2383.031 & 0.132 & 0.065 \\
\hline 6 & 0.93 & 569.198 & 0.04 & 0.01 & 6 & 0.916 & 667.225 & 0.062 & 0.017 & 6 & 0.418 & 2927.271 & 0.088 & 0.041 \\
\hline 7 & 1.03 & 615.664 & 0.07 & 0.04 & 7 & 0.869 & 687.071 & 0.051 & 0.017 & 7 & 0.395 & 1840.021 & 0.251 & 0.113 \\
\hline 8 & 0.92 & 499.564 & 0.06 & 0.03 & 8 & 0.896 & 734.648 & 0.049 & 0.013 & 8 & 0.391 & 2764.211 & 0.057 & 0.025 \\
\hline 9 & 0.91 & 480.534 & 0.07 & 0.02 & 9 & 1.186 & 1319.727 & 0.041 & 0.007 & 9 & 0.413 & 2179.016 & 0.231 & 0.113 \\
\hline 10 & 0.84 & 471.670 & 0.06 & 0.02 & 10 & 0.901 & 666.827 & 0.074 & 0.021 & 10 & 0.403 & 3203.954 & 0.046 & 0.017 \\
\hline 11 & 0.86 & 410.182 & 0.06 & 0.03 & 11 & 0.883 & 573.871 & 0.065 & 0.019 & 11 & 0.349 & 2240.729 & 0.034 & 0.008 \\
\hline \multicolumn{5}{|c|}{ Femoral artery } & \multicolumn{5}{|c|}{ Medial descending aorta } & \multicolumn{5}{|c|}{ Distal descending aorta } \\
\hline 1 & 0.254 & 467.110 & 0.057 & 0.008 & 1 & 0.825 & 584.212 & 0.085 & 0.014 & 1 & 0.809 & 1339.021 & 0.027 & 0.000 \\
\hline 2 & 0.287 & 868.784 & 0.029 & 0.000 & 2 & 0.868 & 851.551 & 0.060 & 0.019 & 2 & 0.831 & 1749.407 & 0.046 & 0.016 \\
\hline 3 & 0.275 & 633.869 & 0.024 & 0.000 & 3 & 0.859 & 699.499 & 0.085 & 0.022 & 3 & 0.821 & 1501.760 & 0.030 & 0.000 \\
\hline 4 & 0.289 & 710.867 & 0.039 & 0.007 & 4 & 0.838 & 650.488 & 0.069 & 0.029 & 4 & 0.818 & 1506.283 & 0.029 & 0.000 \\
\hline 5 & 0.275 & 557.173 & 0.056 & 0.015 & 5 & 0.833 & 661.712 & 0.055 & 0.026 & 5 & 0.822 & 1464.471 & 0.031 & 0.002 \\
\hline 6 & 0.278 & 705.508 & 0.031 & 0.000 & 6 & 0.871 & 738.137 & 0.079 & 0.029 & 6 & 0.829 & 1568.171 & 0.028 & 0.000 \\
\hline 7 & 0.264 & 665.543 & 0.032 & 0.007 & 7 & 0.828 & 702.763 & 0.068 & 0.039 & 7 & 0.848 & 1511.557 & 0.084 & 0.040 \\
\hline 8 & 0.287 & 775.969 & 0.018 & 0.000 & 8 & 0.877 & 949.096 & 0.025 & 0.000 & 8 & 0.826 & 1076.808 & 0.234 & 0.113 \\
\hline 9 & 0.287 & 758.730 & 0.034 & 0.000 & 9 & 0.870 & 727.716 & 0.084 & 0.035 & 9 & 0.824 & 1702.527 & 0.024 & 0.003 \\
\hline 10 & 0.299 & 835.167 & 0.040 & 0.000 & 10 & 0.875 & 949.631 & 0.030 & 0.002 & 10 & 0.828 & 1511.236 & 0.093 & 0.044 \\
\hline 11 & 0.314 & 836.095 & 0.021 & 0.000 & 11 & 0.833 & 595.933 & 0.068 & 0.037 & 11 & 0.441 & 81.464 & 5.546 & 0.261 \\
\hline \multicolumn{15}{|c|}{ Proximal descending aorta } \\
\hline 1 & 0.888 & 681.721 & 0.041 & 0.006 & 5 & 0.916 & 857.673 & 0.039 & 0.003 & 9 & 0.876 & 739.620 & 0.057 & 0.023 \\
\hline 2 & 0.902 & 805.161 & 0.058 & 0.025 & 6 & 0.879 & 765.712 & 0.053 & 0.016 & 10 & 0.903 & 915.101 & 0.039 & 0.003 \\
\hline 3 & 0.852 & 698.828 & 0.035 & 0.007 & 7 & 0.876 & 689.512 & 0.065 & 0.021 & 11 & 0.863 & 620.584 & 0.067 & 0.032 \\
\hline 4 & 0.885 & 739.959 & 0.036 & 0.004 & 8 & 0.856 & 646.061 & 0.058 & 0.027 & & & & & \\
\hline
\end{tabular}

TABLE III

MEAN AND STANDARD DEVIATION FOR EACH LOCATION.

\begin{tabular}{l|cccc} 
& $r_{0}[\mathrm{~cm}]$ & $E h[\mathrm{mmHg} \mathrm{cm}]$ & $\tau_{\epsilon}[\mathrm{sec}]$ & $\tau_{\sigma}[\mathrm{sec}]$ \\
\hline Ascending aorta (S1) & $0.94 \pm 0.06$ & $531.30 \pm 72.22$ & $0.07 \pm 0.01$ & $0.02 \pm 0.01$ \\
Proximal desc. aorta (S2) & $0.88 \pm 0.02$ & $741.00 \pm 89.50$ & $0.05 \pm 0.01$ & $0.02 \pm 0.01$ \\
Medial desc. aorta (S3) & $0.85 \pm 0.02$ & $737.34 \pm 127.43$ & $0.06 \pm 0.02$ & $0.02 \pm 0.01$ \\
Distal desc. aorta (S4) & $0.83 \pm 0.01$ & $1493.12 \pm 186.78$ & $0.06 \pm 0.07$ & $0.02 \pm 0.04$ \\
Femoral artery (S5) & $0.28 \pm 0.02$ & $710.44 \pm 123.96$ & $0.03 \pm 0.01$ & $0.00 \pm 0.01$ \\
Brachiocephalic trunk (S6) & $0.89 \pm 0.10$ & $680.11 \pm 227.07$ & $0.07 \pm 0.03$ & $0.02 \pm 0.01$ \\
Carotid artery (S7) & $0.41 \pm 0.03$ & $2721.11 \pm 482.20$ & $0.11 \pm 0.07$ & $0.05 \pm 0.04$
\end{tabular}

In this study we have only one state variable, namely the cross-sectional area $A(t)$ and four parameters $r_{0}, E h, \tau_{\epsilon}$, and $\tau_{\sigma}$. The cross-sectional area is found as a solution to a differential equation, that can be obtained by rewriting Equation (3) as

$$
\frac{d A}{d t}=\frac{2}{E h \tau_{\epsilon} / r_{0} \sqrt{A_{0} / A^{3}}}\left(\frac{E h}{r_{0}}\left(\sqrt{\frac{A_{0}}{A}}-1\right)+p+\tau_{\sigma} \frac{d p}{d t}\right)=2 \frac{f}{g},
$$

where

$$
f=\frac{E h}{r_{0}}\left(\sqrt{\frac{A_{0}}{A}}-1\right)+p+\tau_{\sigma} \frac{d p}{d t}, \quad g=\frac{E h \tau_{\epsilon}}{r_{0}} \sqrt{\frac{A_{0}}{A^{3}}} .
$$



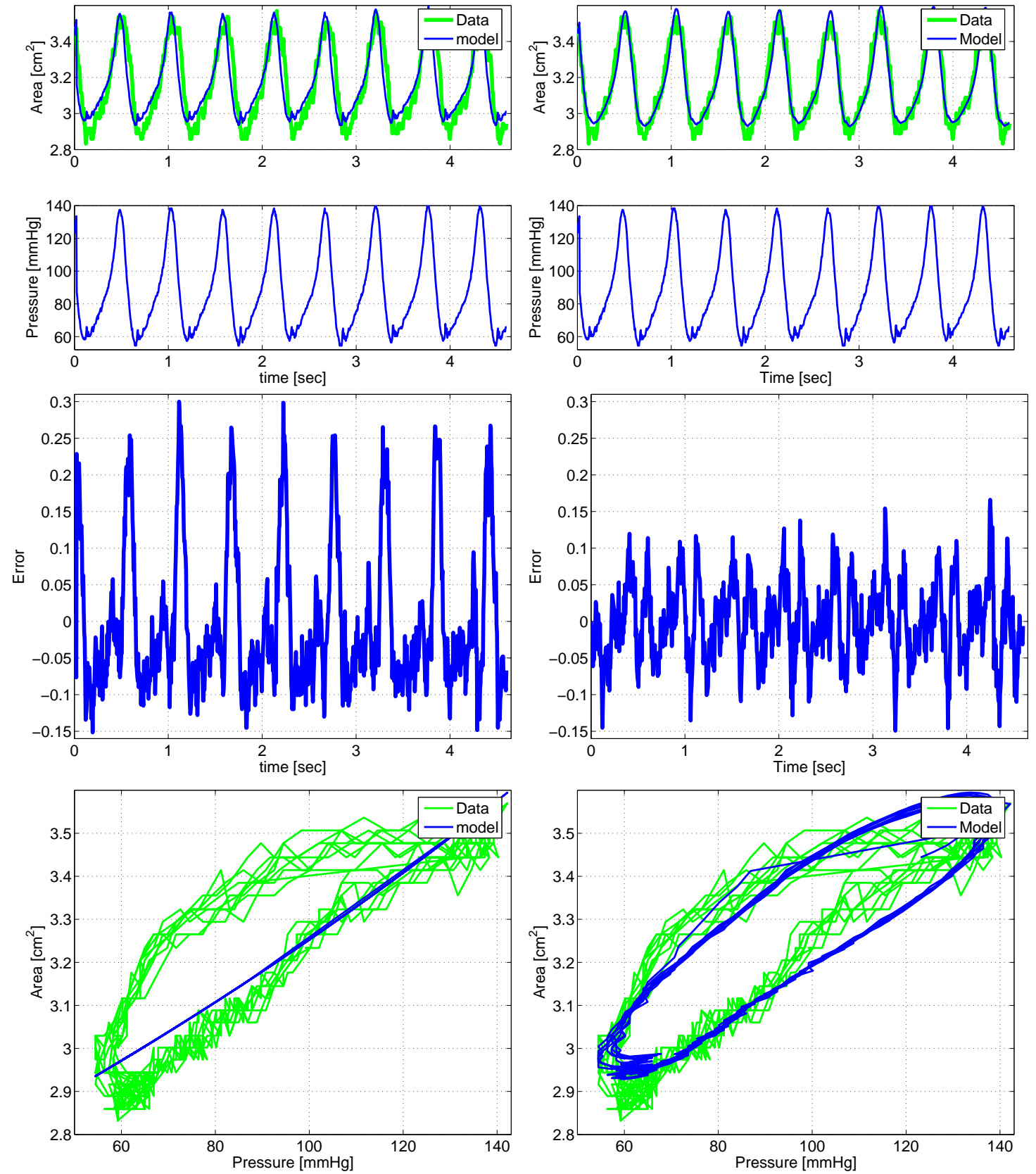

Fig. 4. Top graphs show elastic (left) and viscoelastic (right) models as a function of time, the center row shows the error computed as $\left|A(t)-a_{j}\right|$ for the elastic (left) and the viscoelastic (right) models, and the bottom graphs show hysteresis effects for the elastic model (left) and the viscoelastic model (right).

Given the system, four sensitivity equations can be derived for $\partial A / \partial r_{0}, \partial A / \partial E h, \partial A / \partial \tau_{\epsilon}$, and $\partial A / \partial \tau_{\sigma}$. To determine these sensitivities $\partial A / \partial \theta_{j}$, we differentiate the differential equation with respect to each parameter as

$$
\frac{d}{d t} \frac{\partial A}{\partial \theta_{j}}=\frac{\partial}{\partial \theta_{j}} \frac{d A}{d t}
$$

Here, we assumed that the partial derivatives commute. This is guaranteed by Clairaut's theorem, which states that if continuous second partial derivatives exist at a point, they commute at that point. These four sensitivity equations can thus be solved simultaneously with Equation (11). Using this formulation, 
the sensitivity equations can be found by

$$
\frac{d}{d t} \frac{\partial A}{\partial \theta_{j}}=2\left[g \frac{\partial f}{\partial \theta_{j}}-f \frac{\partial g}{\partial \theta_{j}}\right] / g^{2}
$$

where

$$
\begin{aligned}
\frac{\partial f}{\partial r_{0}} & =-\frac{E h}{r_{0}^{2}}\left(\sqrt{\frac{A_{0}}{A}}-1\right)+\frac{E h}{r_{0}}\left(\sqrt{\frac{\pi}{A}}-\sqrt{\frac{A_{0}}{A^{3}}}\right) / 2 \frac{d A}{d r_{0}} \\
\frac{\partial g}{\partial r_{0}} & =-\frac{3 E h \tau_{\epsilon}}{2} \sqrt{\frac{\pi}{A^{5}}} \frac{d A}{d r_{0}} \\
\frac{\partial f}{\partial E h} & =\frac{1}{r_{0}}\left(\sqrt{\frac{A_{0}}{A}}-1\right)-\frac{E h}{2 r_{0}} \sqrt{\frac{A_{0}}{A^{3}}} \frac{d A}{d E h} \\
\frac{\partial g}{\partial E h} & =\frac{\tau_{\epsilon}}{r_{0}} \sqrt{\frac{A_{0}}{A^{3}}}-\frac{3 E h \tau_{\epsilon}}{2 r_{0}} \sqrt{\frac{A_{0}}{A^{5}}} \frac{d A}{d E h} \\
\frac{\partial f}{\partial \tau_{\epsilon}} & =-\frac{E h}{2 r_{0}} \sqrt{\frac{A_{0}}{A^{3}}} \frac{d A}{d \tau_{\epsilon}} \\
\frac{\partial g}{\partial \tau_{\epsilon}} & =\frac{E h}{r_{0}} \sqrt{\frac{A_{0}}{A^{3}}}-\frac{3 E h \tau_{\epsilon}}{2 r_{0}} \sqrt{\frac{A_{0}}{A^{5}}} \frac{d A}{d \tau_{\epsilon}} \\
\frac{\partial f}{\partial \tau_{\sigma}} & =\frac{d p}{d t}-\frac{E h}{2 r_{0}} \sqrt{\frac{A_{0}}{A^{3}}} \frac{d A}{d \tau_{\sigma}} \\
\frac{\partial g}{\partial \tau_{\sigma}} & =-\frac{3 E h \tau_{\epsilon}}{2 r_{0}} \sqrt{\frac{A_{0}}{A^{5}}} \frac{d A}{d \tau_{\sigma}} .
\end{aligned}
$$

The corresponding relative sensitivities are given by

$$
\frac{\partial A}{\partial E h} \frac{E h}{A}, \quad \frac{\partial A}{\partial r_{0}} \frac{r_{0}}{A}, \quad \frac{\partial A}{\partial \tau_{\epsilon}} \frac{\tau_{\epsilon}}{A}, \quad \frac{\partial A}{\partial \tau_{\sigma}} \frac{d \tau_{\sigma}}{A} .
$$

A typical solution (evaluated at the optimized parameter values $\theta=\hat{\theta}^{n}$ ) for the relative sensitivity equations is shown in Fig. 5. Results from this figure shows that the sensitivities are periodic functions that oscillate relative to the periodicity in the data. To rank the sensitivities we used a max norm computing max relative sensitivities as

$$
\tilde{S}\left(\theta_{j}\right)=\left(\max _{t} \frac{\partial A(t)}{\partial \theta_{j}} \frac{\theta_{j}}{A}\right) .
$$

For the system of sensitivity equations we obtained:

$$
\max \left(\frac{\partial A}{\partial r_{0}} \frac{r_{0}}{A}\right), \quad \max \left(\frac{\partial A}{\partial E h} \frac{E h}{A}\right), \quad \max \left(\frac{\partial A}{\partial \tau_{\epsilon}} \frac{\tau_{\epsilon}}{A}\right), \quad \max \left(\frac{\partial A}{\partial \tau_{\sigma}} \frac{\tau_{\sigma}}{A}\right) .
$$

For sheep 1, these max sensitivities are given by $\left[\tilde{S}\left(r_{0}\right)=2.64 ; \tilde{S}(E h)=0.64 ; \tilde{S}\left(\tau_{\epsilon}\right)=0.087 ; \tilde{S}\left(\tau_{\sigma}\right)=\right.$ 0.042]; i.e., the most sensitive parameter with respect to the change in $A(t)$ is $r_{0}$ and the least sensitive parameter is $\tau_{\sigma}$, the stress relaxation constant.

Results discussed above all compare the model to the data assuming that the model is valid within one dataset. Another use of the sensitivity analysis is to study and compute the standard errors as a mean of computing confidence intervals for each parameter within a dataset and to be able to predict how well the model perform.

In order to compute standard errors and confidence intervals, we used standard asymptotic theory from statistics. We assumed that each dataset predicting vessel area contains $n$ scalar longitudinal observations, which can be represented by the statistical model

$$
\tilde{A}_{j}=f_{j}\left(\theta_{0}\right)+\epsilon_{j}, \quad j=1,2, \ldots n,
$$



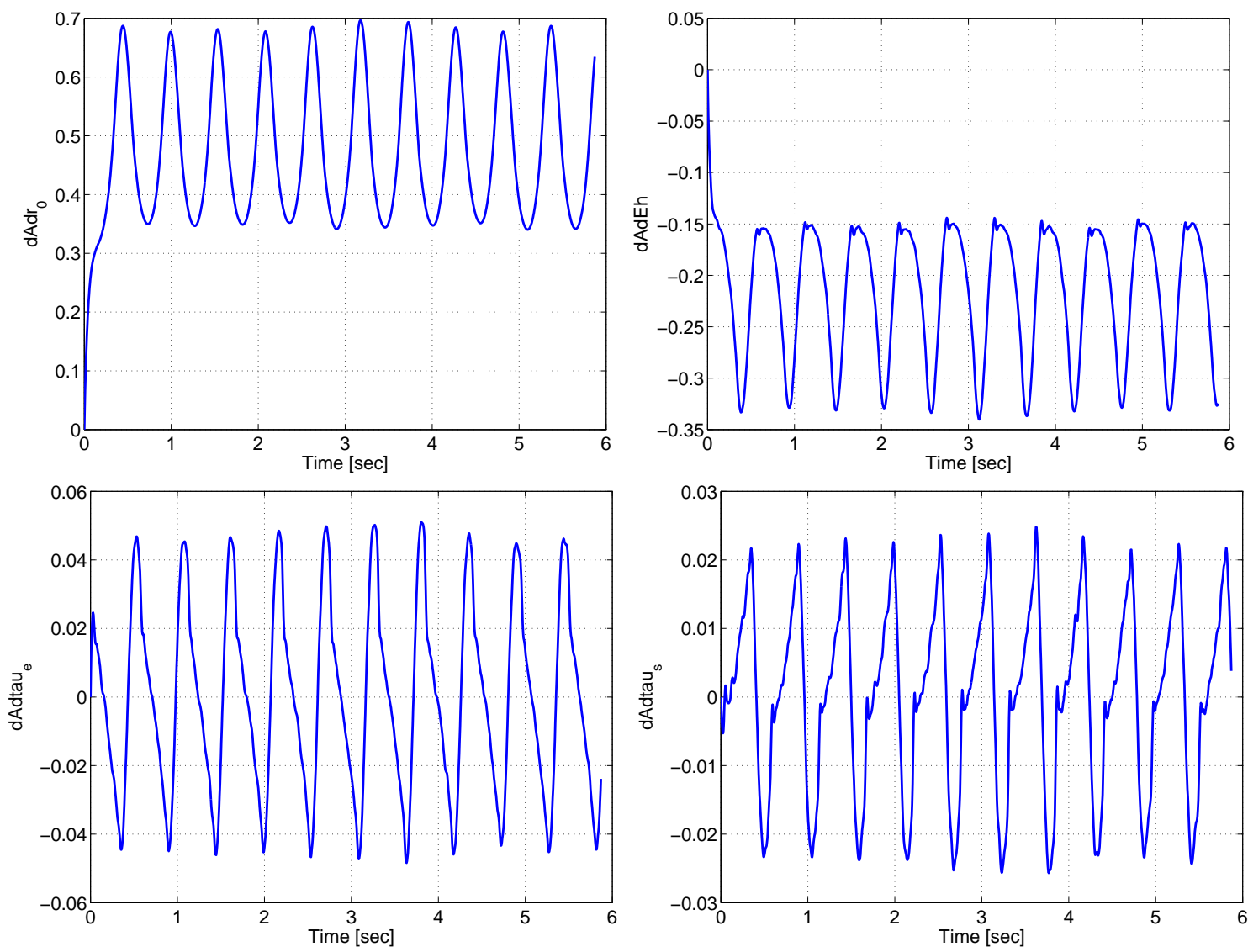

Fig. 5. Relative sensitivity of $A(t)$ with respect to the parameters $d A / d E h, d A / d r_{0}, d A / \tau_{\epsilon}, d A / d \tau_{\sigma}$, respectively. These sensitivities are evaluated at the optimized parameters $\hat{\theta}^{n}$.

where $f_{j}$ is the model for the observations and $\theta_{0} \in \mathbb{R}^{M}$ is a set of theoretical true parameter values for $r_{0}, E h, \tau_{\sigma}$, and $\tau_{\epsilon}$. Furthermore, we assumed that measurement errors $\epsilon_{j}, j=1,2, \ldots, n$, are independent identically distributed (i.i.d.) random variables with mean $E\left[\epsilon_{j}\right]=0$ and constant variance $\operatorname{var}\left[\epsilon_{j}\right]=\sigma_{0}^{2}$, where $\sigma_{0}^{2}$ is unknown.

We consider estimation of parameters using an ordinary least squares (OLS) approach as described above. Thus we seek to use the area data $a=\left\{a_{j}\right\}$ for the observation process $\left\{\tilde{A}_{j}\right\}$ with the model to seek a value $\hat{\theta}^{n}$ that minimizes

$$
J_{n}(\theta)=\Sigma_{j=1}^{n}\left|f_{j}(\theta)-a_{j}\right|^{2} .
$$

This definition differs from Equation (8) where the least squares differences were weighted by $1 / n$, where $n$ is the number of observations; this only serves to scale the residual values.

Under the assumption that state variables and corresponding dynamics used to describe the model are continuously differentiable and certain regularity assumptions on how the observations are made, the standard nonlinear regression approximation theory [43], [44], [45], [46] for asymptotic (as $n \rightarrow \infty$ ) distributions can be invoked. This theory states that the sampling distribution $\hat{\theta}^{n}(\tilde{A})$ for the estimate $\hat{\theta}^{n}$, where $\tilde{A}=\left\{\tilde{A}_{j}\right\}_{j=1}^{n}$, is approximately a $M$-multivariate Gaussian with mean $E\left[\hat{\theta}^{n}(\tilde{A})\right] \approx \theta_{0}$ and covariance

$$
\operatorname{cov}\left[\hat{\theta}^{n}(\tilde{A})\right] \approx \Sigma_{0}=\sigma_{0}^{2}\left[\chi^{T}\left(\theta_{0}\right) \chi\left(\theta_{0}\right)\right]^{-1} .
$$


Here $\chi(\hat{\theta})=F_{\theta}(\theta)$ is the $n \times M$ sensitivity matrix, which has the form

$$
\chi=\left(\begin{array}{cccc} 
& \frac{\partial A}{\partial r_{0}}\left(t_{1}\right) \frac{\partial A}{\partial E h}\left(t_{1}\right) & \frac{\partial A}{\partial \tau_{\epsilon}}\left(t_{1}\right) & \frac{\partial A}{\partial \tau_{T}}\left(t_{1}\right) \\
\frac{\partial A}{\partial r_{0}}\left(t_{2}\right) \frac{\partial A}{\partial E h}\left(t_{2}\right) & \frac{\partial A}{\partial \tau_{\epsilon}}\left(t_{2}\right) & \frac{\partial A}{\partial \tau_{\sigma}}\left(t_{2}\right) \\
\ldots & \cdots & \ldots & \ldots \\
\frac{\partial \ddot{A}}{\partial E h}\left(t_{n}\right) & \frac{\partial \ddot{A}}{\partial r_{0}}\left(t_{n}\right) & \frac{\partial \dot{A}}{\partial \tau_{\sigma}}\left(t_{n}\right) & \frac{\partial \dot{A}}{\partial \tau_{\epsilon}}\left(t_{n}\right)
\end{array}\right) .
$$

In other words, for $n$ large, the sampling distribution approximately satisfies

$$
\hat{\theta}_{O L S}^{n}(\tilde{A}) \sim \mathcal{N}_{M}\left(\theta_{0}, \sigma_{0}^{2}\left[\chi^{T}\left(\theta_{0}\right) \chi\left(\theta_{0}\right)\right]^{-1}\right):=\mathcal{N}_{M}\left(\theta_{0}, \Sigma_{0}\right) .
$$

For the viscoelastic model the function $f_{j}(\theta)$ is sufficiently simple to derive analytical expressions for the components of $\partial A / \partial \theta$. However, $\theta_{0}$ and $\sigma_{0}$ are not known, hence they must be approximated to calculate $\Sigma_{0}=\sigma_{0}^{2}\left[\chi^{T}\left(\theta_{0}\right) \chi\left(\theta_{0}\right)\right]^{-1}$. For this we follow standard practice and use the approximation

$$
\Sigma_{0} \approx \Sigma\left(\hat{\theta}^{n}\right)=\hat{\sigma}\left[\chi^{T}\left(\hat{\theta}^{n}\right) \chi\left(\hat{\theta}^{n}\right)\right]^{-1},
$$

where $\hat{\theta}^{n}$ is the parameter estimate obtained, and the approximation $\hat{\sigma}^{2}$ to $\sigma_{0}^{2}$ is given by [46]

$$
\sigma_{0}^{2} \approx \hat{\sigma}^{2}=\frac{1}{n-4} \sum_{i=1}^{n}\left|a_{j}-f_{j}\left(\hat{\theta}^{n}\right)\right|^{2} .
$$

Standard errors to be used in confidence intervals calculations are thus given by (see [46])

$$
S E_{k}\left(\hat{\theta}^{n}\right)=\sqrt{\Sigma_{k k}\left(\hat{\theta}^{n}\right)}
$$

which embodies uncertainty in the estimates for $n$ large. Finally, the confidence intervals (at the $100(1-\alpha) \%$ level) for the estimated parameters, are given by

$$
\left[\hat{\theta}_{k}^{n}-t_{1-\alpha / 2} S E_{k}\left(\hat{\theta}^{n}\right), \hat{\theta}_{k}^{n}+t_{1-\alpha / 2} S E_{k}\left(\hat{\theta}^{n}\right)\right],
$$

where

$$
P\left\{\hat{\theta}_{k}^{n}-t_{1-\alpha / 2} S E_{k}\left(\hat{\theta}^{n}\right)<\theta_{0 k}<\hat{\theta}_{k}^{n}+t_{1-\alpha / 2} S E_{k}\left(\hat{\theta}^{n}\right)\right\}=1-\alpha,
$$

for $\alpha \in[0,1]$ and $t_{1-\alpha / 2} \in \mathbb{R}_{+}$. For a $95 \%$ confidence intervals, the critical value $t_{1-\alpha / 2}$ is computed from the Student's $t$ distribution $t^{n-4}$ with $n-4$ degrees of freedom. The value of $t_{1-\alpha / 2}$ is determined by $P\left\{T>t_{1-\alpha / 2}\right\}=\alpha / 2$ where $T \sim t^{n-4}$. Given that in the data set $n>40$, the degrees of freedom was approximated to $\infty$. Thus $t_{1-\alpha / 2} \approx 1.96$.

Below we have computed confidence intervals using all available data (10 cardiac cycles) from sheep 1. To compute the confidence interval, we used optimized parameters given in Table II. For this dataset the error is

$$
J=\sum_{i}^{n}\left|a_{j}-A\left(t_{j}\right)\right|^{2}=5.860,
$$

and the variance is

$$
\sigma_{0}^{2} \approx \hat{\sigma}^{2}=\frac{1}{n-4} \sum_{i=1}^{n}\left|a_{j}-A\left(t_{j}\right)\right|^{2}=0.005 .
$$

In Fig. 6 we plotted variance as a function of time and as a function of area. Using these values we computed the covariance matrix

$$
\Sigma_{0} \approx \Sigma\left(\hat{\theta}^{n}\right)=\sigma^{2}\left[\chi^{T}\left(\hat{\theta}^{n}\right) \chi\left(\hat{\theta}^{n}\right)\right]^{-1}=\left(\begin{array}{rrrr}
71.638 & 0.024 & -0.041 & -0.029 \\
0.024 & 0.000^{*} & -0.000 & -0.000 \\
-0.041 & -0.000 & 0.000^{*} & 0.000 \\
-0.029 & -0.000 & 0.000 & 0.000^{*}
\end{array}\right)
$$


and the standard error

$$
S E_{k}\left(\hat{\theta}^{n}\right)=\sqrt{\Sigma_{k k}\left(\hat{\theta}^{n}\right)}=\left(\begin{array}{c}
8.464 \\
0.003 \\
0.005 \\
0.004
\end{array}\right) .
$$

Finally, the confidence computed using Equation (15) gives

$$
\begin{aligned}
C I_{E h} & =[461.94,495.12] \\
C I_{r 0} & =[0.904,0.916] \\
C I_{\tau_{\epsilon}} & =[0.043,0.063] \\
C I_{\tau_{\sigma}} & =[0.014,0.030] .
\end{aligned}
$$
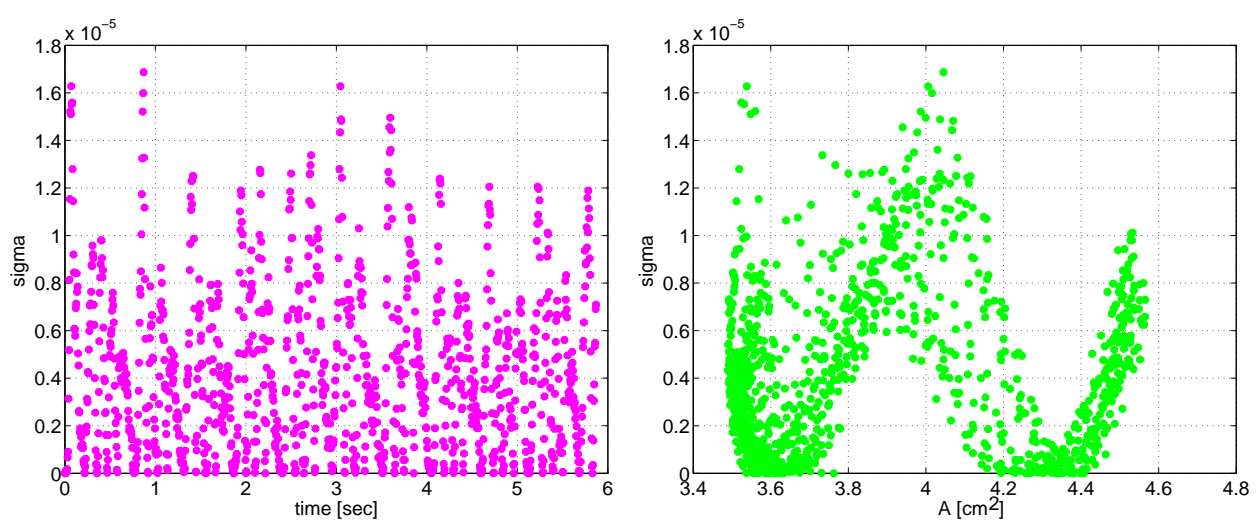

Fig. 6. The left panel depicts the residual sum of squares as a function of time and the right panel depicts the residual sum of squares as a function of the model response (the area $A(t)$ ).

\section{RESULTS}

As discussed in the previous section our results clearly shows that the viscoelastic model is significantly better than the purely elastic model. The elastic model cannot account for hysteresis which is clearly observed in the data. Results from our statistical analysis and our sensitivity studies indicate that we can identify all model parameters. Hence, we proceed to discuss computational results obtained from the model.

From calculation of $p$-values (see Table IV) we observe that it is easy to distinguish the caliber (radius) for each vessel. We used the diastolic diameter as the initial value for the unstretched radius, but this parameter was identified during optimization. Another conclusion we can make is that the ascending aorta, the carotid artery, and the distal descending artery, have significantly different values for $E h$ than the other arteries. For example, the femoral artery, as well as the medial and proximal descending aorta all have p-values $>0.05$. We also observed that the relaxation factors for the more distal femoral and carotid arteries are significantly different from the more proximal vessels. This is reasonable since the latter vessels should be stiffer than the major conduction arteries. Another interesting aspect to study is the notion discussed in most physiology books (e.g., Guyton [40]) that arteries gets stiffer as they get smaller. While stiffness has been studied using purely elastic models (see e.g. [15], [16]), it is not clear how the relative stiffness (i.e., $E h / r_{0}$ ) changes when viscoelastic properties are also considered. Furthermore, results discussed in previous studies were compiled across species and were from a number of different experiments. Given the large dataset presented from sheep, we were able to study how stiffness related to the radius of the artery according to the different locations of the vessel. As shown in Fig. 7 it is clear that 
TABLE IV

P-VALUES COMPARING EACH LOCATION FOR EACH PARAMETER.

\begin{tabular}{|l|cccccc||c|cccccc|}
\hline$r_{0}$ & $\mathrm{~B}$ & $\mathrm{C}$ & $\mathrm{D}$ & $\mathrm{F}$ & $\mathrm{M}$ & $\mathrm{P}$ & $\mathrm{Eh}$ & $\mathrm{B}$ & $\mathrm{C}$ & $\mathrm{D}$ & $\mathrm{F}$ & $\mathrm{M}$ & $\mathrm{P}$ \\
\hline $\mathrm{A}$ & 0.21 & 0.00 & 0.00 & 0.00 & 0.00 & 0.01 & $\mathrm{~A}$ & 0.05 & 0.00 & 0.00 & 0.00 & 0.00 & 0.00 \\
$\mathrm{~B}$ & - & 0.00 & 0.04 & 0.00 & 0.21 & 0.71 & $\mathrm{~B}$ & - & 0.00 & 0.00 & 0.70 & 0.47 & 0.41 \\
$\mathrm{C}$ & - & - & 0.00 & 0.00 & 0.00 & 0.00 & $\mathrm{C}$ & - & - & 0.00 & 0.00 & 0.00 & 0.00 \\
$\mathrm{D}$ & - & - & - & 0.00 & 0.10 & 0.02 & $\mathrm{D}$ & - & - & - & 0.00 & 0.00 & 0.00 \\
$\mathrm{~F}$ & - & - & - & - & 0.00 & 0.00 & $\mathrm{~F}$ & - & - & - & - & 0.62 & 0.50 \\
$\mathrm{M}$ & - & - & - & - & - & 0.00 & $\mathrm{M}$ & - & - & - & - & - & 0.93 \\
\hline \hline$\tau_{\epsilon}$ & $\mathrm{B}$ & $\mathrm{C}$ & $\mathrm{D}$ & $\mathrm{F}$ & $\mathrm{M}$ & $\mathrm{P}$ & $\tau_{\sigma}$ & $\mathrm{B}$ & $\mathrm{C}$ & $\mathrm{D}$ & $\mathrm{F}$ & $\mathrm{M}$ & $\mathrm{P}$ \\
\hline $\mathrm{A}$ & 0.77 & 0.09 & 0.33 & 0.00 & 0.95 & 0.01 & $\mathrm{~A}$ & 0.57 & 0.05 & 0.44 & 0.00 & 0.76 & 0.04 \\
$\mathrm{~B}$ & - & 0.13 & 0.33 & 0.00 & 0.76 & 0.07 & $\mathrm{~B}$ & - & 0.04 & 0.38 & 0.00 & 0.81 & 0.26 \\
$\mathrm{C}$ & - & - & 0.37 & 0.01 & 0.09 & 0.02 & $\mathrm{C}$ & - & - & 0.85 & 0.00 & 0.04 & 0.01 \\
$\mathrm{D}$ & - & - & - & 0.30 & 0.33 & 0.32 & $\mathrm{D}$ & - & - & - & 0.11 & 0.41 & 0.26 \\
$\mathrm{~F}$ & - & - & - & - & 0.00 & 0.01 & $\mathrm{~F}$ & - & - & - & - & 0.00 & 0.00 \\
$\mathrm{M}$ & - & - & - & - & - & 0.06 & $\mathrm{M}$ & - & - & - & - & - & 0.15 \\
\hline
\end{tabular}

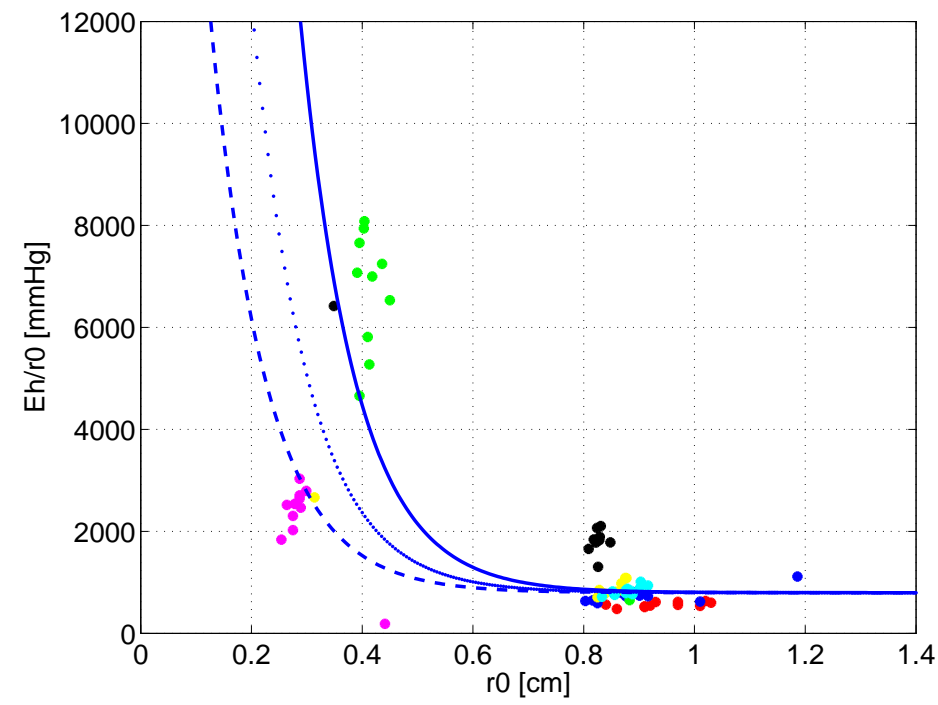

Fig. 7. Stiffness relative to radius as a function of the radius of the unstretched vessel $r_{0}$. Results for each vessel segment are represented by a different dot color.

our studies in sheep support the notion that the arterial wall stiffness, increases with decreasing vessel diameter. To illustrate this finding we have fit an exponentially decreasing function to the corresponding data. Similar to previous work [16], [15], [47] we have used an Equation of the form

$$
\frac{E h}{r_{0}}=k_{1} \exp \left(-k_{2} r_{0}\right)+k_{3} \text {. }
$$

Note that for small arteries (the carotid arteries and the femoral arteries) it is not clear how the graph should be weighted (compare solid, dashed, and dotted lines). From physiology it is known that carotid arteries are less stiff than arteries in other parts of the body, hence, the graph likely should be weighted towards points representing the femoral artery (dashed line).

\section{DISCUSSION}

The goal of this study was to develop a mathematical model that can be used to analyze experimental pressure-area data and to understand how the associated model parameters vary across the large arteries. The goal was not to develop the most sophisticated model, but to come up with a simple model, which for example, could be incorporated into fluid dynamics models. The next question to address is weather 
the physical accuracy of the Kelvin viscoelastic model and the associated four model parameters can be predicted with a high level of confidence. In the following we first discuss some experimental considerations and then discuss limitations and further studies from a modeling perspective.

\section{A. Methodological considerations}

We studied sheep segments, taking into account the recognized similarity between human and ovine cardiovascular systems [48]. We opted for vascular segments, instead of the most used strips or rings [49], [50], since segments were better to reproduce the in vivo hemodynamic conditions, and to preserve the shape and integrity of the vascular wall [33]. The in vitro stretch of the artery segment should be the same with respect to in vivo condition. In our experiment, an arterial segment of $6 \mathrm{~cm}$ long was accurately measured with a caliper and marked with two suture stitches. All arteries were excised at the level of the suture stitches and, after vascular removal, were mounted preserving the in vivo length [33], [34], [35].

The technique employed to register pressure and diameter using the in vitro circulation mock has been previously used and validated by members of our group [2], [31], [32], [33], [34], [35]. This methodology allows accurate and reproducible pressure and diameter measurements due to the high frequency and linearity of response of both dimension gauges and pressure transducers. The $200 \mathrm{~Hz}$ sampling rate used in the digitalization of the data was larger than the highest frequency components in the pressure and diameter spectra, enabling signal reconstruction without distortion.

The arterial wall has a nonlinear and frequency-dependent mechanical behavior [2], [32]. Hence, although static analysis allow the indirect evaluation of rate or frequency-dependent mechanical properties (e.g., wall viscosity) [49], [50], an adequate characterization of the functional meaning and contribution of

these properties requires dynamic analysis. Consequently, in order to evaluate the arterial wall mechanical behavior, we performed a dynamic analysis of the arterial wall, submitting the arteries to physiological hemodynamic conditions. On the other hand, taking into account the pressure and frequency rate dependence of the mechanical properties, we performed an isobaric and isofrequency analysis during physiological flow condition for each artery [2], [32].

Diameter and pressure signals was conformed and processed using a single analogic electronic system (System VI Triton Technology San Diego California) resulting in no delay between pressure and diameter signals caused by the electronics circuits. Nevertheless, for a correct use of the pressure-area data or the stress-strain loops to calculate the viscous properties, it is essential that both distension and pressure waves should be recorded simultaneously at the same location, because the existence of misalignment (yet of only a few millimeters) strongly affects the shape of the pressure-distension loop. However, as was mentioned previously [37], and as was performed in previous work by members of our group [31], [33], to calculate wall viscoelasticity based on the method described in the article, the diameter signal is shifted to fit the pressure signal foot, to compensate the overestimation in the time delay corresponding to the distance between sensors.

\section{B. Modeling considerations}

Results from this study indicates excellent agreement between predictions of our theoretical model and the corresponding experimental data. This was evidenced by improvement of the Kelvin viscoelastic model over the elastic model and, in particular, the incorporation of hysteresis into the wall mechanics which lead to a substantial decrease in the computed least squares error $J$. Even though the hysteresis loops exhibited by the Kelvin viscoelastic model more accurately represent the pressure-area data, it is clear that the linear assumption in the Kelvin model is limiting, given the clear nonlinear trends in the data. Nevertheless, the results from this study confidently allow us to pursue extended viscoelastic modeling by studying, in greater depth, the viscoelastic properties of the arterial wall and potential variations in model assumptions with vessel site.

Formulating the hysteresis dynamics in Equations (4)-(7) as we did, using part of the data (i.e., $\left\{p\left(t_{j}\right)\right\}$ ) to reduce the inverse problem involving Equation (8), and then using standard statistical techniques for 
error analysis introduces some modeling and statistical inaccuracies of a nontrivial nature. Indeed we have used interpolated data $\left\{p_{j}\right\}$ in evaluating $s(t)$ in Equation (7) so our model in itself contains measurement error of a type not treated in the standard representation given by Equation (14). This, rather than a simple nonconstant longitudinal variance, is most likely the origin of the somewhat strange "residual versus model" plot shown in Fig. 6. A more proper formulation of the hysteretic dynamics Equation (4) would be of the form

$$
G\left(A(t), p_{t}(\cdot), \theta\right)=0,
$$

where $A(t), s(t), p(t)$ are related through Equations (2) and (3). One should then use the data $\left\{a_{j}, p_{j}\right\}$ for $A(t), p(t)$ in a least squares criterion

$$
J(\theta)=\Sigma\left|\left(a_{j}, p_{j}\right)-\left(A\left(t_{j}, \theta\right), p\left(t_{j}, \theta\right)\right)\right|^{2}
$$

subject to the constants in Equation (16). This leads immediately to a nonstandard least squares problem with associated computational challenges as well as statistical questions that we are pursuing in a continuing effort.

\section{ACKNOWLEDGMents}

This experimental work was partially supported by Programa Desarrollo Ciencias Basicas (PEDECIBA, Uruguay). We thank Mr. Elbio Agote for his valuable assistance during the development of the experiments. The modeling efforts were supported in part by NSF under grant DMS0616597, by the U.S. Air Force Office of Scientific Research under grant AFOSR-FA9550-04-1-0220, and by the Undergraduate Research Office at North Carolina State University. 


\section{REFERENCES}

[1] R. Vito and S. Dixon, "Blood vessel constitutive models - 1995-2002," Ann Rev Biomed Eng, vol. 5, pp. 413-439, 2003.

[2] R. Armentano, J. Barra, J. Levenson, A. Simon, and R. Pichel, "Arterial wall mechanics in conscious dogs. assessment of viscous, inertial, and elastic moduli to characterize aortic wall behaviour," Circ Res, vol. 76, pp. 468-478, 1995.

[3] P. Mangell, T. Lanne, B. Soneson, F. Hansen, and D. Bergqvist, "Regional differences in mechanical properties between major arteries - an experimental study in sheep," Eur J Vasc Endovasc Surg, vol. 12, pp. 189-195, 1996.

[4] M. O'Rourke, "Arterial mechanics and wave reflection with antihypertensive therapy," J Hypertens Suppl, vol. 10, pp. S43-S49, 1992.

[5] S. Glasser, "On arterial physiology, pathophysiology of vascular compliance and cardiovascular disease," Heart Dis, vol. 2, pp. 375-379, 2000.

[6] S. Laurent, P. Lacolley, X. Girerd, P. Boutouyrie, Y. Bezie, and M. Safar, "Arterial stiffening: opposing effects of age- and hypertension-associated structural changes," Can J Pharmacol, vol. 74, pp. 842-849, 1996.

[7] G. London, "Large artery function and alterations in hypertension," J Hypertens Suppl, vol. 13, pp. S35-S38, 1995.

[8] M. O'Rourke and W. Nichols, "Aortic diameter, aortic stiffness, and wave reflection increases with age and isolated systolic hypertension," Hypertension, vol. 45, p. 652, 2005.

[9] A. Veress, D. Vince, P. Anderson, J. Cornhill, E. Herderick, J. Klingensmith, and B. Kuban, "Vascular mechanics of the coronary artery," Z Kardio, vol. 89 Suppl 2, pp. 92-100, 2000.

[10] J. Humphrey, "Mechanics of the arterial wall: Review and directions," Crit Rev Biomed Eng, vol. 2, pp. 1-162, 1995.

[11] R. O. G.A. Holzapfel, T.C. Gasser, "A new constitutive framework for arterial wall mechanics and a comparitive study of material models," J of Elasticity, vol. 61, pp. 1-48, 2000.

[12] M. S. G.A. Holzapfel, T.C.N. Gasser, "A structural model for the viscoelastic behavior of arterial walls: Continuum formulation and finite element simulation," Eur J Mech Solids, vol. 21, pp. 441-463, 2002.

[13] R. Bauer, R. Busse, A. Shabert, Y. Summa, and E. Wetterer, "Separate determination of the pulsatile elastic and viscous forces developed in the arterial wall in vivo," Pflugerrs Arch, vol. 380, pp. 221-226, 1979.

[14] J. Grotberg and O. Jensen, "Biofluid mechanics in flexible tubes," Ann Rev of Fluid Mech, vol. 36, pp. 121-147, 2004.

[15] M. Olufsen, C. Peskin, W. Kim, E. Pedersen, A. Nadim, and J. Larsen, "Numerical simulation and experimental validation of blood flow in arteries with structured tree outflow conditions," Ann Biomed Eng, vol. 28(11), pp. 1281-1299, 2000.

[16] M. Olufsen, "A structurewd tree outflow condition for blood flow in the larger systemic arteries," Am J Physiol, vol. 276, pp. H257-H268, 1999.

[17] A. Quarteroni, M. Tuveri, and A. Veneziani, "Computational vascular fluid dynamics: Problems models, and methods," Comput Vis Sci, vol. 2, pp. 163-197, 2000.

[18] J. Cebral, P. Yim, R. Lohner, O. Soto, and P. Choyke, "Blood flow modeling in carotid arteries with computational fluid dynamics and mr imaging," Acad Radiol, vol. 9, pp. 1286-1299, 2002.

[19] B. Steele, J. Wan, J. Ku, T. Hughes, and C. Taylor, "In vivo validation of a one-dimensional finite-element method for predicting blood flow in cardiovascular bypass grafts," IEEE Trans Biomed Eng, vol. 6, pp. 649-656, 2003.

[20] C. Taylor and M. Draney, "Experimental and computational methods in cardiovascular fluid mechanics," Ann Rev Fluid Mech, vol. 36, pp. 197-231, 2006.

[21] D. Tang, C. Yang, S. Kobayashi, J. Zheng, and R. Vito, "Effect of stenosis asymmetry on blood flow and artery compression: a tree-dimensional fluid-structure interaction model," Ann Biomed Eng, vol. 31, pp. 1182-1193, 2003.

[22] D. Tang, C. Yang, S. Kobayashi, and D. Ku, "Effect of a lipid pool on stress/strain distributions in stenotic arteries: 3-d fluid-structure interactions (fsi) models," J Biomech Eng, vol. 126, pp. 363-370, 2004.

[23] C. Smith, "On the introduction of viscoelasticity into one-dimensional models of arterial blood flow," Eng Phys Astron, vol. 43, pp. 1286-1299, 1982.

[24] S. Canic, C. Hartley, D. Rosenstrauch, J. Tambaca, G. Guidoboni, and A. Mikelic, "Blood flow in compliant arteries: an effective viscoelastic reduced model, numerics, and experimental validation," Ann Biomed Eng, vol. 34, pp. 575-592, 2006.

[25] S. Canic, J. Tambaca, G. Guidoboni, A. Mikelic, C. Hartley, and D. Rosenstrauch, "Modeling viscoelastic behavior of arterial walls and their interaction with pulsatile blood flow," SIAM J Appl Math, vol. 67, pp. 164-193, 2006.

[26] G. Pontrelli, "A mathematical model of flow in a liquid-filled visco-elastic tube," Med Biol Eng Comput, vol. 40, pp. 550-556, 2002.

[27] U. N. R. Council, Guide for the Care and use of Laboratory Animals. Washington, DC: National Academy Press, 1996.

[28] R. Armentano, J. Barra, D. B. Santana, F. Pessana, S. Graf, D. Craiem, L. Brandani, H. Baglio, and R. Sanchez, "Smart damping modulation of carotid wall energetics in human hypertension: effects of ace inhibition," Hypertension, vol. 87, pp. 384-290, 2006.

[29] R. Armentano, D. B. Santana, E. Cabrera, S. Graf, H. Campos, Y. Zocalo, M. Saldias, and I. Alvarez, "An in vitro study of cryopreserved and fresh human arteries: a comparison with eptfe prostheses and human arteries studied non-invasively in vivo," Cryobiology, vol. 52(1), pp. 17-26, 2006.

[30] D. B. Santana, R. Armentano, J. Grignola, D. Craiem, Y. Zocalo, F. Gines, and J. Levenson, "The vascular smooth muscle of great arteries: local control site of arterial buffering function ?" Rev Esp Cardiol, vol. 56(12), pp. 1202-1209, 2003.

[31] D. B. Santana, R. Armentano, D. Craiem, J. Grignola, F. Gines, A. Simon, and J. Levenson, "Smooth muscle role on pulmonary arterial function during acute pulmonary hypertension in sheep," Acta Physiol Scand, vol. 181(3), pp. 359-366, 2004. 
[32] D. B. Santana, J. Barra, J. Grignola, F. Gines, and R. Armentano, "Pulmonary artery smooth muscle activation attenuates arterial dysfunction during acute pulmonary hypertension," J Appl Physiol, vol. 98(2), pp. 605-613, 2005.

[33] D. B. Santana, Y. Zocalo, F. Pessana, R. Armentano, H. Perez-Campos, M. Saldias, and I. Alvarez, "Femoral arteries energy dissipation and filtering function remain unchanged after cryopreservation procedure," Transpl Int, vol. 18(12), pp. 1346-1355, 2005.

[34] E. Cabrera, R. Armentano, F. Pessana, S. Graf, L. Romero, A. Christen, A. Simon, and J. Levenson, "Endothelium-dependent arterial wall tone elasticity modulated by blood viscosity," Am J Physiol, vol. 282, pp. H389-H394, 2002.

[35] Y. Zocalo, F. Pessana, D. B. Santana, and R.Armentano, "Regional differences in veins wall dynamics under arterial hemodynamics conditions: comparison with arteries," Artificial Organs, vol. 30(4), pp. 265-275, 2006.

[36] R. Armentano, J. Barra, F. Pessana, D. Craiem, S. Graf, D. B. Santana, and R. Sanchez, "Smart smooth muscle springdampers. smooth muscle smart filtering helps to more efficiently protect the arterial wall," IEEE Eng Med Biol Mag, vol. 26(1), pp. 62-70, 2007.

[37] A. H. J. Willigers and R. Reneman, "Effects of assessment and processing techniques on the shape of arterial pressuredistension loops," J Vasc Res, vol. 37, pp. 494-450, 2000.

[38] Y. Fung, Biomechanics, Mechanical Properties of Living Tissues. New York, NY: Springer Verlag, 1993.

[39] — Biomechanics, Circulation. New York, NY: Springer Verlag, 1996.

[40] A. Guyton and J. Hall, Textbook of Medical Physiology, 9th ed. Philadelphia, PE: WB Saunders., 1996.

[41] M. Eslami, Theory of Sensitivity in Dynamic Systems: An Introduction. Berlin, Germany: Spring-Verlag, 1994.

[42] P. Frank, Introduction to Sensitivity Theory. New York, NY: Academic Press, 1978.

[43] M. Davidian and D. Giltinan, Nonlinear Models for Repeated Measurements Data. London, UK: Chapman \& Hall, 1998.

[44] R. Jennrich, "Asymptotic properties of non-linear least squares estimators," Ann Math Statist, vol. 40, pp. 663-643, 1969.

[45] G. Seber and C. Wild, Nonlinear Regression. New York, NY: John Wiley \& Sons, Inc, 1989.

[46] G. Casella and R. Berger, Statistical Inference. California: Duxbury, 2002.

[47] J. Ottesen, M. Olufsen, and J. Larsen, Applied Mathematical Models in Human Physiology. Philadelphia, PE: SIAM, Monographs on Math Model Comput, 2004.

[48] T. Kohler and T. Kirkman, "Dialysis access failure: A sheep model of rapid stenosis," J Vasc Surg, vol. 30(4), pp. 744-751, 1999.

[49] F. Silver, P. Snowhill, and D. Foran, "Mechanical behavior of vessel wall: a comparative study of aorta, vena cava, and carotid artery," Ann Biomed Eng, vol. 31(7), pp. 793-803, 2003.

[50] D. Mavrilas and T. Tsapikouni, "Dynamic mechanical properties of arterial and venous grafts used in coronary bypass surgery," J Mech Med Biol, vol. 2(3-4), pp. 1-9, 2002. 